\title{
Magnetic fields in A-type stars associated with X-ray emission
}

\author{
C. Schröder ${ }^{1}$, S. Hubrig ${ }^{2}$, and J. H. M. M. Schmitt ${ }^{1}$ \\ 1 Hamburger Sternwarte, Gojenbergsweg 112, 21029 Hamburg, Germany \\ e-mail: cschroeder@hs.uni-hamburg.de \\ 2 European Southern Observatory, Casilla 19001, Santiago 19, Chile
}

Received 30 October 2007 / Accepted 25 February 2008

\section{ABSTRACT}

\begin{abstract}
A common explanation for the observed X-ray emission of A-type stars is the presence of a hidden late-type companion. While this assumption can be shown to be correct in some cases, a number of lines of evidence suggests that low-mass companions cannot be the correct cause for the observed activity in all cases. A model explains the X-ray emission for magnetic Ap/Bp stars, focusing on the A0p star IQ Aur. In this paper we test whether this theoretical model is able to explain the observed X-ray emission. We present the observations of 13 A-type stars that have been associated with X-ray emission detected by ROSAT. To determine the mean longitudinal magnetic field strength we measured the circular polarization in the wings of the Balmer lines using FORS1. Although the emission of those objects that possess magnetic fields fits the prediction of the Babel and Montmerle model, not all $\mathrm{X}$-ray detections are connected to the presence of a magnetic field. Additionally, the measured magnetic fields do not correlate with the X-ray luminosity. Accordingly, the magnetically confined wind shock model cannot explain the X-ray emission from all the presented stars.
\end{abstract}

Key words. stars: magnetic fields - stars: activity - X-rays: stars - stars: early-type

\section{Introduction}

One of the outstanding discoveries of X-ray astronomy is the detection of X-ray emission from essentially all late-type main sequence stars (cf. see the compilation by Schmitt \& Liefke 2004, and references therein). For hot stars X-ray luminosity scales with the stars' bolometric luminosity, while for late-type stars $\mathrm{X}$-ray emission is intimately linked to stellar rotation and convection. The role of magnetic fields - if any - in the X-ray emission of early-type stars is unclear. Almost immediately after the detection of X-ray emission from early-type stars (Seward et al. 1979; Harnden et al. 1979), Lucy \& White (1980) and Lucy (1982) proposed a phenomenological model, in which the X-ray emission is generated from instabilities in these stars' radiatively driven winds (Feldmeier et al. 1997; Owocki \& Cohen 1999); an example of this kind of X-ray producing mechanism is the prototypical O-type star $\zeta$ Pup (Kramer et al. 2003). An alternative wind shock model was introduced by Babel \& Montmerle (1997), who assume large scale magnetic structures confining the (still radiatively driven) wind; the wind shock model can be brought into very good agreement with the observations (Gagné et al. 2005) for the magnetic O-type star $\Theta^{1}$ Ori C. On the other hand, the X-ray emission from late-type stars is usually interpreted as a scaled-up version of solar X-ray emission, where the emitting hot plasma is believed to be confined by coronal magnetic fields. Indeed, convection and rotation have been shown to be the central ingredients of the magnetic dynamos thought to be ultimately responsible for the observed activity phenomena in the Sun and other late-type stars (see Schmitt et al. 1995, and references therein).

In this scenario, A-type stars ought to be devoid of any $\mathrm{X}$-ray emission. The winds of such stars should be either absent or be weak at best. However, contrary to expectations some A-type stars do in fact show X-ray emission. About $15 \%$ of all bright A-type stars can be identified with X-ray sources found in the ROSAT All Sky Survey or ROSAT pointed observations (Schröder \& Schmitt 2007). Specifically, out of 1966 bright A-type stars $\left(m_{v}<6.5\right), 312$ were detected as X-ray sources, with 194 of these detections occurring in known binary systems. Subdivisions of these stars into Ap, Am and giant A-type stars gives detection rates similar to the overall sample. The usual explanation of these 194 "unexpected" X-ray detections is to attribute their X-ray emission to the presence of low mass companions, and in the 118 A-type stars without known companions such companions are postulated to exist. It is of course relatively easy to "hide" a low mass companion of, say, spectral type M in the vicinity of a bright A-type star. Further, A-type stars are by comparison young and thus an hypothetical late-type companion should be quite X-ray luminous, so that the observed X-ray luminosities of such A-type stars are consistent with X-ray luminosities observed for young $\mathrm{M}$ stars and even T-Tauri stars. In some specific cases, the correctness of the above described lowmass companion scenario can be explicitly shown. For example, in the case of the totally eclipsing binary $\alpha \mathrm{CrB}$, a total X-ray eclipse is found at the time of optical secondary minimum, when the A-type star in the system is located in the front of the X-ray emitting G-type star (Schmitt \& Kürster 1993), thus proving that indeed essentially all the X-ray flux from this system does come from the G-type star.

Assuming this "companion hypothesis" to be correct, a few strange findings, which have been accumulated over the years, remain to be explained (summarized by Schmitt et al. 2005). First, in quite a few cases (Berghöfer \& Schmitt 1994; Hubrig et al. 2001; Stelzer et al. 2006a) high angular resolution data of (visual) binary systems show X-ray emission from both system components, implying that further components need to be introduced to explain the observed A-type star X-ray emission. Next, the star with the largest measured magnetic field of $35000 \mathrm{G}$ 
is Babcock's star HD 215441 and its spectral type A0Vp suggests neither the presence of convection nor of a strong wind. Yet Babcock's star is an X-ray source (Czesla \& Schmitt 2007). $\mathrm{X}$-ray emission and in particular X-ray flaring has been observed from the (extremely) peculiar B-type star $\sigma$ Ori E with a surface magnetic field of $10 \mathrm{kG}$ (Groote \& Schmitt 2004), yet no companion is known. Two dimensional MHD simulations from ud-Doula et al. (2006) suggest that these flares are caused by centrifugal breakout of the magnetically confined line-driven wind. Further, X-ray emission has been observed not only from Babcock's star, but also stars with the highest measured magnetic fields as listed in the compilation by Bychkov et al. (2003). An orbital modulation of the X-ray emission has been observed for the magnetic stars $\Theta$ Ori C (Gagne et al. 1997) as well as HD 133880 (Czesla \& Schmitt, in preparation). Finally, a survey for X-ray emission for the magnetic stars listed in the catalog by Bychkov et al. (2003) showed a total of 23 stars detected as X-ray sources identifiable with A-type stars with large magnetic fields (Schmitt et al. 2005). It appears to be extremely contrived to attribute the observed X-ray emission in all these cases to hitherto unseen late-type companions. Rather the existence of a large scale magnetic field seems to be necessary for the production of these "unexpected" X-ray emissions. With the so-called magnetically confined wind-shock model by Babel \& Montmerle (1997) a theoretical framework has been constructed to explain the observed high energy emissions. In the last decade, significant progress has been made, for example by Donati et al. (2002), ud-Doula \& Owocki (2002), Gagné et al. (2005) and Townsend et al. (2007), to describe and simulate the interactions between the line-driven winds and the magnetosphere of hot stars like $\sigma$ Ori $\mathrm{E}$ and $\Theta$ Ori $\mathrm{C}$. The predictions of the dynamical models used by these authors are in good agreement with the observations, and therefore motivated us to search for possible evidence for magnetically confined wind shocks in X-ray emitting A-type stars. Specifically, in this paper we try to test whether the detection of X-ray emission is directly related to the presence of a large scale magnetic field, as suggested by the wind-shock model. Additionally, we check if the measured magnetic field strengths are correlated to the X-ray luminosity of the star.

We present the observations and the data reduction technique in Sect. 2. Section 3 is divided into the calculations of the magnetic fields, a verification of the magnetically confined windshock model for those stars that have been detected at a $3 \sigma$ level and some comments on those stars. We summarize and discuss our data in Sect. 4.

\section{Observations and data reduction}

The observations reported in this paper were carried out on August 28th 2006 with FORS1 at the VLT Kueyen. This multimode instrument is equipped with polarization analyzing optics comprising super-achromatic half-wave and quarter-wave phase retarder plates, and a Wollaston prism with a beam divergence of $22^{\prime \prime}$ in standard resolution mode. Two different grisms were used, the GRISM 600B and the GRISM 1200B, which cover all Balmer lines from $\mathrm{H}_{\beta}$ to the Balmer jump. 12 stars have were observed with the GRISM 600B and the corresponding spectral resolution of 2000, and three stars with the GRISM 1200B at a resolving power of $R \sim 4000$. GRISM 1200B does not include all hydrogen lines down to the Balmer jump $(\sim 3650 \AA)$. The blue limit of the spectra is $3885 \AA$. Since we had only one observing night, we decided to observe only the two most promising targets with GRISM 1200B.
During the calibration runs in daytime two retarder waveplate setups with angles of $+45^{\circ}$ and $-45^{\circ}$ were used. The calibration itself was achieved by combining the science spectra with the corresponding calibration spectra of the same retarder waveplate angle. Ordinary and extraordinary beams were independently calibrated with the corresponding beams of the reference spectrum. By examining the differences between the circular polarizations observed in the red and blue wings of the hydrogen line profiles it is possible to measure the mean longitudinal field $\left\langle B_{z}\right\rangle$ (Landstreet 1982) by using the formula

$\frac{V}{I}=-\frac{g_{\mathrm{eff}} e \lambda^{2}}{4 \pi m_{\mathrm{e}} c^{2}} \frac{1}{I} \frac{\mathrm{d} I}{\mathrm{~d} \lambda}\left\langle B_{z}\right\rangle$.

In (1) $V$ denotes the Stokes parameter measuring the the circular polarization, $I$ denotes the intensity in the unpolarized spectrum, $g_{\mathrm{eff}}$ is the effective Landé factor, $e$ denotes the electron charge, $\lambda$ the wavelength, $m_{\mathrm{e}}$ the electron mass, $c$ the speed of light, $\mathrm{d} I / \mathrm{d} \lambda$ is the derivative of Stokes $I$, and $\left\langle B_{z}\right\rangle$ is the mean longitudinal field. The $V / I$ values were calculated according to:

$\frac{V}{I}=\frac{1}{2}\left\{\left(\frac{f^{\mathrm{o}}-f^{\mathrm{e}}}{f^{\mathrm{o}}+f^{\mathrm{e}}}\right)_{\alpha=-45}-\left(\frac{f^{\mathrm{o}}-f^{\mathrm{e}}}{f^{\mathrm{o}}+f^{\mathrm{e}}}\right)_{\alpha=+45}\right\}$

where $\alpha$ is the position angle of the retarder waveplate, which was rotated by $90^{\circ}\left(+45^{\circ},-45^{\circ},-45^{\circ},+45^{\circ}\right.$ etc. $)$ for the different exposures to reduce any cross-talk effect. $f^{\circ}$ and $f^{\mathrm{e}}$ denote the ordinary and the extraordinary beams, which have been summed to obtain the Stokes $I$ values. For each star we took three to four pairs of exposures with two orientations of the retarder waveplate, resulting in six to eight exposures for each object. A more detailed description of this method has been given by Bagnulo et al. (2002) and Hubrig et al. (2003, 2004a).

\section{Results}

\subsection{Stellar sample and magnetic field detections}

The stars in our sample were selected from a list of bright A-type stars, which can be associated with a X-ray source compiled by Schröder \& Schmitt (2007). Out of 84 stars known as single or resolved binary stars a subsample of 13 stars (excluding the standard star HD 201601) was selected taking into account observational boundary conditions. In Table 1 we provide the basic data of the observed stars as well as the results of our magnetic field measurements. The columns in Table 1 give the HD number, the HR number, the visual magnitude, the spectral type, the X-ray luminosity, the mean longitudinal magnetic field determined by all spectral lines, the mean longitudinal magnetic field extracted from the hydrogen lines given in Table 1, the averaged quadratic magnetic field whenever available as listed in the Bychkov catalog of stellar effective magnetic fields and in the last column remarks about the individual stars. The values of the averaged quadratic magnetic field given in Col. 11 are calculated by

$\left\langle B_{\mathrm{e}}\right\rangle=\left(\frac{1}{n} \sum_{i=1}^{n} B_{\mathrm{e} i}^{2}\right)^{\frac{1}{2}}$

where $B_{\mathrm{e} i}$ is the $i$ th measurement of the effective magnetic field, and $n$ is the total number of observations for a given star. The averaged quadratic errors are calculated accordingly.

The low-resolution FORS1 spectropolarimeter is usually used to measure circular polarization in the wings of strong and broad spectral lines like Balmer lines or strong $\mathrm{He}$ or $\mathrm{Ca}$ lines. One of the reasons for this is that weaker and narrower metal 
Table 1. Basic data and measured magnetic fields for the stars from the GRISM 600 in the upper part of the table and the GRISM 1200 in the lower part. Stars with a longitudinal magnetic field at a $3 \sigma$ level are indicated in bold face.

\begin{tabular}{|c|c|c|c|c|c|c|c|c|c|c|c|}
\hline $\begin{array}{c}\text { HD } \\
\text { GRISM } 600\end{array}$ & Other id. & $V$ & Sp. type & $\begin{array}{l}\text { Dist } \\
{[\mathrm{pc}]}\end{array}$ & $\begin{array}{l}\log L_{\mathrm{x}} \\
{[\mathrm{erg} / \mathrm{s}]}\end{array}$ & $\log L_{\mathrm{x}} / L_{\mathrm{bol}}$ & $\begin{array}{r}v \sin (i) \\
{\left[\mathrm{km} \mathrm{s}^{-1}\right]}\end{array}$ & $\begin{array}{l}\left\langle B_{z}\right\rangle \text { all } \\
{[\mathrm{G}]}\end{array}$ & $\begin{array}{c}\left\langle B_{z}\right\rangle \text { hydr. } \\
{[\mathrm{G}]}\end{array}$ & $\begin{array}{c}\left\langle B_{\mathrm{e}}\right\rangle \text { lit. }^{1} \\
{[\mathrm{G}]}\end{array}$ & Rem. \\
\hline 147084 & $o \mathrm{Sco}$ & 4.6 & A4II/III & $361 \pm 99$ & 29.89 & -0.91 & 23 & $-79 \pm 35$ & $-133 \pm 46$ & $170 \pm 98$ & \\
\hline 159217 & $\sigma$ Ara & 4.6 & $\mathrm{~A} 0 \mathrm{~V}$ & $118 \pm 10$ & 29.66 & -2.30 & - & $67 \pm 52$ & $62 \pm 54$ & & \\
\hline 159312 & HR 6539 & 6.5 & A0V & $104 \pm 10$ & 29.96 & -2.94 & - & $128 \pm 73$ & $241 \pm 80$ & & \\
\hline 163336 & HR 6681 & 5.9 & $\mathrm{~A} 0 \mathrm{~V}$ & $76 \pm 6$ & 29.49 & -3.63 & 60 & $2 \pm 43$ & $20 \pm 60$ & & \\
\hline 172555 & HR 7012 & 4.8 & A5IV-V & $29 \pm 1$ & 28.85 & -4.89 & - & $27 \pm 34$ & $31 \pm 56$ & & disk \\
\hline 174240 & HR 7085 & 6.2 & A1V & $176 \pm 25$ & 29.30 & -3.23 & 102 & $113 \pm 54$ & $98 \pm 64$ & & \\
\hline 182761 & HR 7384 & 6.3 & A0V & $123 \pm 11$ & 30.08 & -2.69 & 186 & $22 \pm 50$ & $-35 \pm 58$ & & \\
\hline 186219 & HR 7498 & 5.4 & A4III & $42 \pm 1$ & 29.34 & -4.42 & - & $56 \pm 29$ & $58 \pm 62$ & & \\
\hline 201601 & $\gamma \mathrm{Equ}$ & 4.7 & A9p & $35 \pm 1$ & $<28.47$ & - & 10 & $-1121 \pm 34$ & $-1085 \pm 42$ & (Sect. 3.3) & std \\
\hline 215789 & $\epsilon$ Gru & 3.5 & A3V & $40 \pm 1$ & 28.00 & -4.75 & - & $29 \pm 71$ & $37 \pm 85$ & & disk \\
\hline 217186 & HR 8738 & 6.3 & A1V & $96 \pm 8$ & 28.95 & -4.04 & 60 & $97 \pm 53$ & $93 \pm 66$ & & \\
\hline 224361 & HR 9060 & 6.0 & A1IV & $63 \pm 2$ & 29.49 & -3.89 & - & $-63 \pm 60$ & $12 \pm 83$ & & \\
\hline 224392 & $\eta$ Tuc & 5.0 & A1V & $49 \pm 1$ & 29.00 & -4.17 & 187 & $134 \pm 51$ & $89 \pm 60$ & & disk \\
\hline \multicolumn{12}{|l|}{ GRISM 1200B } \\
\hline 147084 & $o \mathrm{Sco}$ & 4.6 & A4II/III & $361 \pm 99$ & 29.89 & -0.91 & 23 & $-48 \pm 15$ & $-87 \pm 36$ & $170 \pm 98$ & \\
\hline 148898 & $\omega \mathrm{Oph}$ & 4.4 & A7p & $54 \pm 2$ & 29.34 & -3.76 & 60 & $122 \pm 29$ & $186 \pm 60$ & $249 \pm 169$ & \\
\hline 201601 & $\gamma \mathrm{Equ}$ & 4.7 & A9p & $35 \pm 1$ & $<28.47$ & - & 10 & $-1200 \pm 12$ & $-1136 \pm 27$ & (Sect. 3.3) & std \\
\hline
\end{tabular}

${ }^{1}$ Catalogue of stellar effective magnetic fields (Bychkov et al. 2003).

lines appear unresolved at the low spectral resolution achievable with FORS1 $(R \sim 2000-4000)$ and the magnetic fields determined from all metal lines frequently appear to have lower strengths when compared to the fields measured using intrinsically broad hydrogen lines. The accuracy of the magnetic field measurements depends on the spectral type and the complexity of the spectra of the studied targets, on their rotational velocity, but of course also on the strength of the measured magnetic field. Our experience from a study of a large sample of magnetic and non-magnetic Ap and Bp stars and Herbig Ae stars (Hubrig et al. 2004b, 2006) shows that magnetic fields measured from Balmer lines only or using also all metal lines are in good agreement with each other for weak magnetic fields of the order of 300-400 G and less. For stronger fields the difference between both field measurements becomes larger than the measurement uncertainties with a tendency for magnetic fields to be larger when measured from hydrogen lines. The probable explanation for such a behavior is that Eq. (1) applied to metal lines is only valid in the weak field regime. Since the measured magnetic fields in this study are very weak, we present in Table 2 both measurements, using hydrogen lines and all metal lines.

Out of the 13 stars studied (excluding the standard star HD 201601), 8 show signatures of magnetic fields. The three stars HD 147084, HD 148898 and HD 159312 have field measurements above the $3 \sigma$ level in all or the hydrogen lines, while previous measurements of B-fields were below the $2 \sigma$ level for HD 147084 and HD 148898 and the field measurement for HD 159312 is entirely new. The stars with a longitudinal magnetic field at at least a $3 \sigma$ level are indicated in bold face in Table 1. The stars HD 174240 and HD 224392 are detected above the $B_{z}>2 \sigma$ level in all lines, the stars HD 186219 and HD 217186 just below the $B_{z}>2 \sigma$, while the Balmer line only detections have less significance, therefore follow-up measurements for these objects are highly desirable before any definite conclusions with respect to the presence of larger magnetic fields can be drawn. For the rest of our sample stars, i.e. HD 159217, HD 163336, HD 172555, HD 182761, HD 215789 and HD 224361, we do not claim any magnetic field detections. Thus in summary, out of 13 observed stars, we have 3 detections, 4 candidates for magnetic field detections and 6 non-detections.

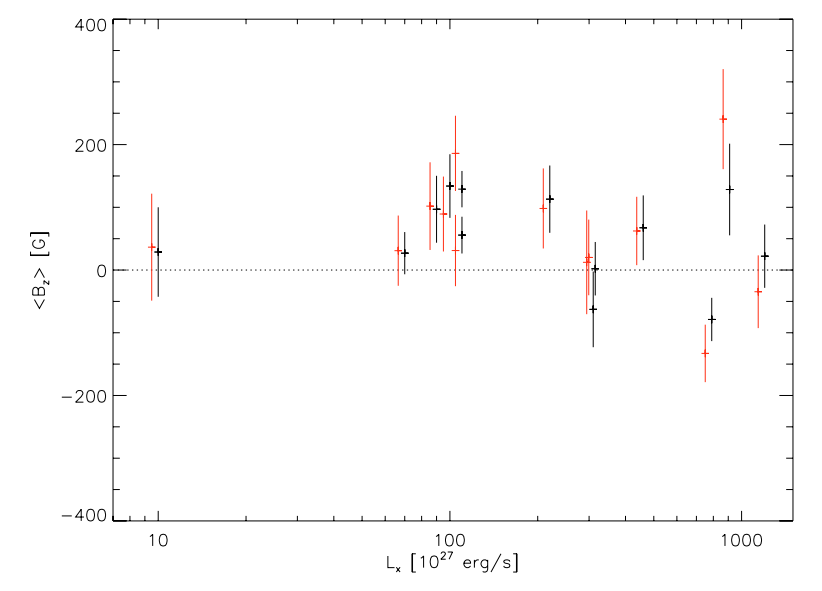

Fig. 1. Mean longitudinal magnetic field measurements plotted over $L_{\mathrm{x}}$ of the associated X-Ray source. Black symbols show the data measured by all lines, red data the values extracted from the hydrogen lines. For purposes of clarity, the red data points are shifted to the left by a few percent.

Figure 1 shows the magnetic field measurements, including the $1 \sigma$ error bars, plotted over the X-ray luminosity of the associated X-ray source. The black symbols represent the values extracted from all available spectral lines, while the red data points show the magnetic field calculated from the hydrogen lines. In order to increase the clarity of the plot, we shifted the hydrogen lines measurements by a few percent. As the plot shows, no sign of a correlation between the X-ray luminosity and the magnetic field strength has been found. We note that because of the strong dependence of the longitudinal field on the rotational aspect, its usefulness to characterize actual field strength distributions is rather limited (Hubrig et al. 2007a). If the magnetic axis is tilted to the rotation axis, as is often the case, the component of the magnetic field parallel to the line of sight changes with the rotation phase. This can be overcome, at least in part, by additional observations to sample various rotation phases, hence various aspects of the field. 
The mean longitudinal magnetic field is the average over the stellar hemisphere visible at the time of observation of the component of the magnetic field parallel to the line of sight, weighted by the local emergent spectral line intensity. It is diagnosed from the slope of a linear regression of $V / I$ versus the quantity $-g_{\mathrm{eff}} \Delta \lambda_{z} \lambda 2 \frac{1}{I} \frac{\mathrm{d} I}{\mathrm{~d} \lambda}\left\langle B_{z}\right\rangle+V_{0} / I_{0}$. This procedure is described in detail by Bagnulo et al. (2002) and Hubrig et al. (2004a). Our experience with a study of a large sample of magnetic and nonmagnetic Ap and Bp stars revealed that this regression technique is very robust and that detections with $B_{z}>3 \sigma_{z}$ result only for stars possessing magnetic fields (Hubrig et al. 2006).

\subsection{Disk contamination in $\mathrm{H}_{\alpha}, \mathrm{H}_{\beta}$ and $\mathrm{Ca}$ II $\mathrm{H}$ and $\mathrm{K}$}

In a few cases, namely HD 172555, HD 215789 and HD 224392, the presence of a circumstellar disk might contaminate the $\mathrm{H}_{\alpha}$, $\mathrm{H}_{\beta}$ and CaII $\mathrm{H}$ and $\mathrm{K}$ lines. The contamination also could affect $\mathrm{H}_{\gamma}, \mathrm{H}_{\delta}$ and the higher Balmer lines, but it is weaker than in $\mathrm{H}_{\alpha}$ and $\mathrm{H}_{\beta}$. To test the influence of this line contamination on the magnetic field measurements we calculated the magnetic fields in two ways: one calculation was done using all available spectral lines in the given spectral area, the other method was using just the hydrogen lines (see Table 1). Comparing the results for the two different line sets, there is no influence on the magnetic field measurements. Two disk stars, HD 172555 and HD 215789, have no magnetic field. HD 224392 shows signs of magnetic fields at the $2 \sigma$ level. In all these measurements the error bars are smaller in the calculations using all available spectral lines. This is no surprise, since the larger number of data points for the calculation of the magnetic field due to the larger number of measured spectral lines results in a lower error value. A systematic difference between the two line sets in respect of the presence of a disk has not been found.

\subsection{Notes on individual stars}

- HD 147084: is an A4 giant star associated with an X-ray source at a distance of 5 arcsec from the optical position of the star. Observations of this object have been performed in the PSPC pointing mode, resulting in an X-ray luminosity of $7.9 \times 10^{29} \mathrm{erg} / \mathrm{s}$. The nature of this object is unclear. If it is a member of Sco OB2, which has an age of around $30 \mathrm{Myr}$ and includes some pre-main sequence objects (Eggen 1998), it might be a laggard Herbig Ae star. However, Whittet (1988) argues against the presence of an infra-red excess, which would be expected for a Herbig Ae star. The Hipparcos and Tycho Catalogues list two entries with distances of $360 \pm 100 \mathrm{pc}$ and $200 \pm 90 \mathrm{pc}$ for HD 147084. The first measurement would question the association of this object with Sco OB2, which lies at a distance of roughly $150 \mathrm{pc}$; the second value would support membership. Admittedly, at such distances, the Hipparcos data have substantial errors. Another possibility would be that o Sco is a Ceph type radial pulsator. If this object is a massive star and member of Sco OB2, it might have left the main sequence after $30 \mathrm{Myr}$ and became a Cepheid-type variable. The magnetic field measurements revealed a weak field of $-79 \pm 35 \mathrm{G}$ in all lines and $-133 \pm 46 \mathrm{G}$ in the hydrogen lines in the GRISM 600B observations and $-48 \pm$ $15 \mathrm{G}$ in all lines and $-87 \pm 36 \mathrm{G}$ in the hydrogen lines in the GRISM 1200B observations. Distinct Zeeman features are visible in the $\mathrm{Ca}$ II $\mathrm{H}$ and $\mathrm{K}$ lines (see Fig. 2). Similar features have been discovered in the spectra of the Herbig Ae star HD 31648 (Hubrig et al. 2007b), which is shown in Fig. 3. In the Catalogue of stellar effective magnetic fields (Bychkov et al. 2003) HD 147084 is recorded with $170 \pm$ 98 G. According to Levato et al. (1987), the radial velocity variations with an amplitude of $50 \mathrm{~km} \mathrm{~s}^{-1}$ may be due to intrinsic motions of the atmosphere. We therefore do not necessarily associate this radial velocity variations with the presence of a late-type companion.

- HD 148898: is a chemical peculiar single star of spectral type A7p. The X-ray source, which is separated from the optical position of the A-type star by 8 arcsec, possesses an X-ray luminosity of $1.1 \times 10^{29} \mathrm{erg} / \mathrm{s}$. Bychkov et al. (2003) list this star with a magnetic field of $248.6 \pm 169.4 \mathrm{G}$ based on the measurements of Borra \& Landstreet (1980). Our calculations resulted in a magnetic field of $122 \pm 29 \mathrm{G}$ for all lines and $186 \pm 60 \mathrm{G}$ for the hydrogen lines, therefore exceeding the $3 \sigma$ level. The Stokes $I$ and $V / I$ spectra are presented in Fig. 4. In May 2003 Hubrig et al. observed this star with the GRISM 600B and found a magnetic field of $221 \pm$ $38 \mathrm{G}$ for all lines and $175 \pm 64 \mathrm{G}$ for the hydrogen lines.

- HD 159217: This A0V star has an associated X-ray source listed in the ROSAT All-Sky Survey catalog. The separation from the optical position of the star is about 8 arcsec and the X-ray luminosity is $4.6 \times 10^{29} \mathrm{erg} / \mathrm{s}$. No indicator for the presence of a hidden companion has been found. The magnetic field measurements yield no detection. The Stokes $I$ and $V / I$ spectra for this star are presented in Fig. 5.

- HD 159312: is a single A0V star with a magnetic field of $128 \pm 73 \mathrm{G}$ measured with all spectral lines and $241 \pm 80 \mathrm{G}$ in the hydrogen lines (see Fig. 5). The longitudinal field is a first detection at a $3 \sigma$ level (see Fig. 1). This star has been detected in the ROSAT All Sky Survey and in the pointed observations with the PSPC instrument. While the X-ray source listed in the RASS catalog has a separation of 5 arcsec from the optical position of the star, the PSPC pointings have a larger offset of $20^{\prime \prime}$ between the X-ray and optical positions. The larger offset in the pointing observation is most likely due to location of the source near the PSPC entrance rib, while the good agreement between RASS and optical position is probably a chance coincidence. No indications for a hidden late type companion have been found.

- HD 163336: is an AOV star associated with an X-ray source separated by less than 3 arcsec from the optical position of the star with an X-ray luminosity of $3.1 \times 10^{29} \mathrm{erg} / \mathrm{s}$ in the HRI observations. No signs of a hidden companion have been found. Our measurements showed no significant magnetic field, but close inspection shows an increased signal in the Stokes $V / I$ spectrum at the positions of the $\mathrm{H}_{\delta}$ and $\mathrm{H}_{\epsilon}$ line (see Fig. 6). Hence this star might be a possible candidate for further observations.

- HD 172555: has a spectral type of A5IV-V and is one of the three stars in our sample with a debris disk. The star is only detected in the ROSAT All-Sky Survey with a separation of 27 arcsec between the optical position and the X-ray source. With a X-ray luminosity of $7 \times 10^{28} \mathrm{erg} / \mathrm{s}$ this object is one of the fainter sources in our sample. A M0 dwarf is positioned at a separation of $71 \mathrm{arcsec}$. The X-ray emission from the M-type star and that from the A-type star are elongated in a way that they form an area of X-ray emission covering the positions of the two stars. In a Chandra observation from March 2004, the M dwarf was clearly detected but no X-ray source was found at the position of the A star. The upper limit for a thermal spectrum with a temperature of $0.9 \mathrm{keV}$ is $5.7 \times 10^{26} \mathrm{erg} / \mathrm{s}$. The non-detection of HD 172555 suggests 

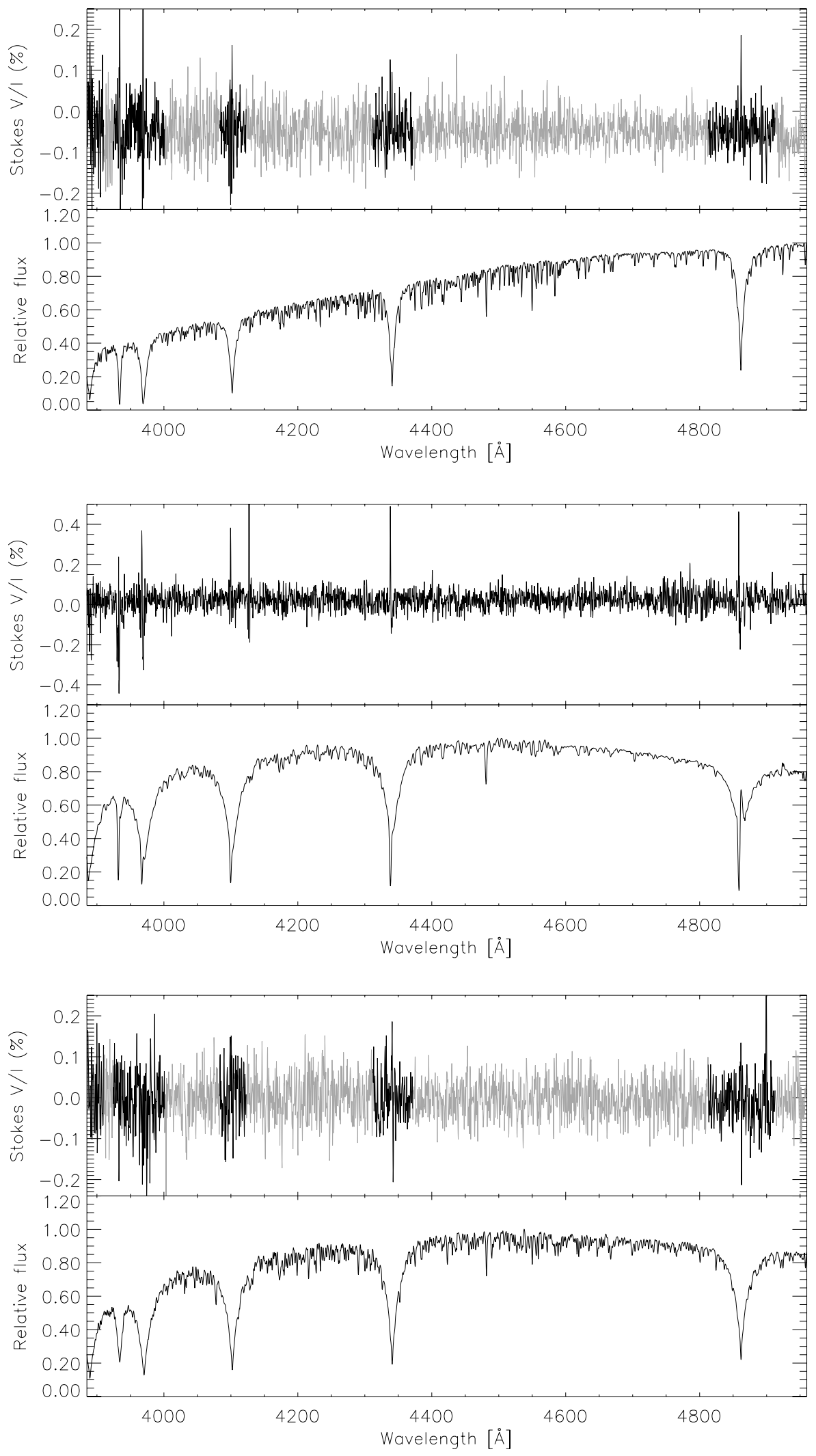

Fig. 2. Stokes $V / I$ spectrum and relative flux of the A4 giant star HD 147084. The black areas in the Stokes $V$ spectrum indicate the regions used for determination of the magnetic fields from Balmer lines. The data shown have been observed with the GRISM 1200B. For comparison see the Herbig Ae/Be star HD 31648 in Fig. 3.

Fig. 3. For comparison with Fig. 2; the Stokes $V / I$ spectrum and relative flux of the Herbig Ae/Be star star HD 31648 observed by Hubrig et al. (2007b). Strong Zeeman features can be found in all Balmer and the CaII $\mathrm{K}$ line.

Fig. 4. Stokes $V / I$ spectrum and relative flux of the A7p star HD 148898. The black areas in the Stokes $V$ spectrum indicate the regions used for determination of the magnetic fields from Balmer lines. that the RASS detection might be an artifact. Radial velocity measurements revealed no variations. The magnetic field measurement yielded no detection.

- HD 174240: The associated X-ray source of this A1V star was observed in the PSPC pointing mode. Its luminosity is $2.2 \times 10^{29} \mathrm{erg} / \mathrm{s}$. The absence of radial velocity variations reduces the possibility for a hidden companion. A longitudinal magnetic field was measured at the $2 \sigma$ level.

- HD 182761: This AOV star is associated with an X-ray source listed in the ROSAT All-Sky Survey catalog. The separation from the optical position of the star is about 25 arcsec and the X-ray luminosity is $1.2 \times 10^{30} \mathrm{erg} / \mathrm{s}$, making this 

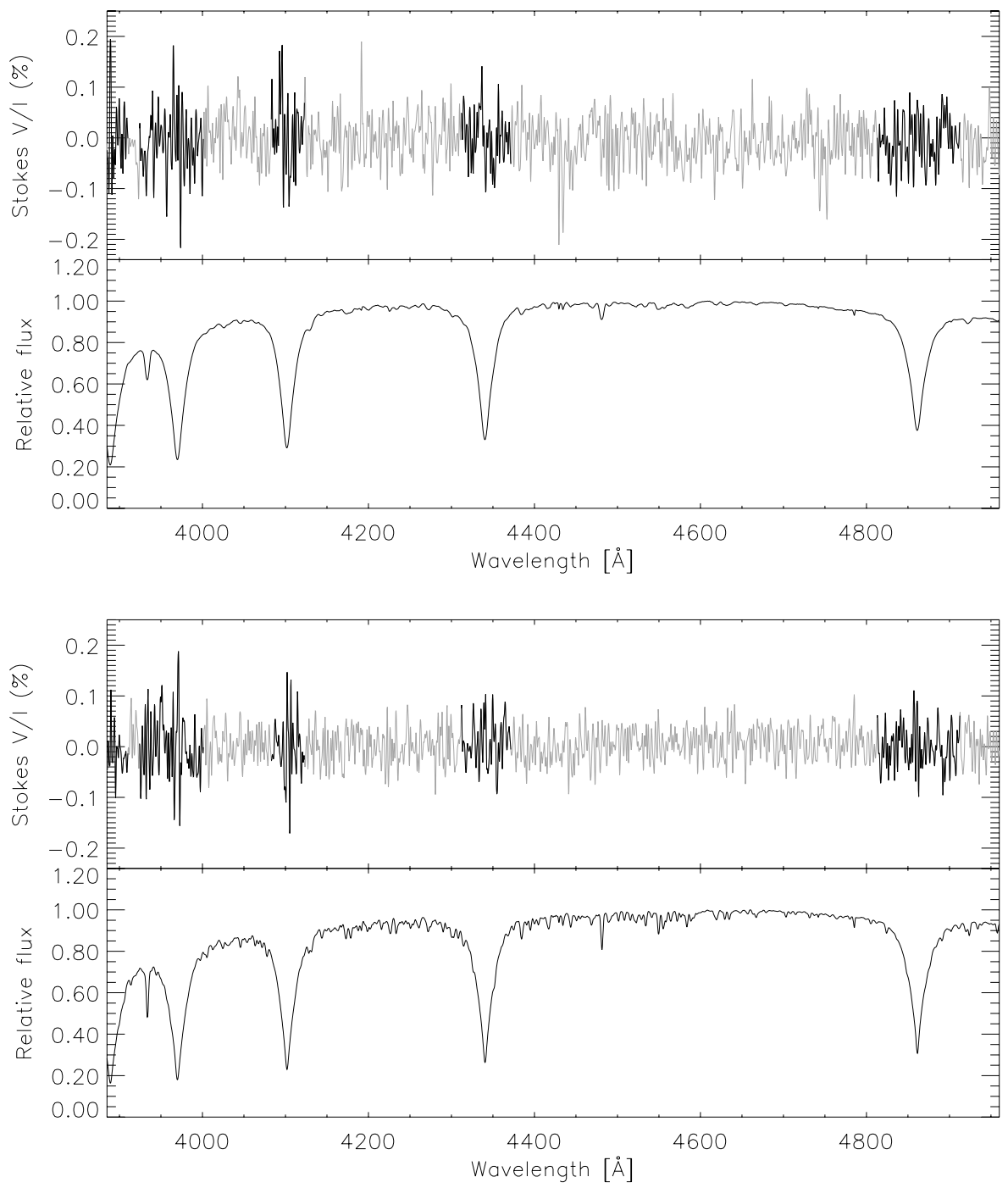

Fig. 5. Stokes $V / I$ spectrum and relative flux of the A0V star HD 159312. The black areas in the Stokes $V$ spectrum indicate the regions used for determination of the magnetic fields from Balmer lines.
Fig. 6. Stokes $V / I$ spectrum and relative flux of the A0V star HD 163336. The black areas in the Stokes $V$ spectrum indicate the regions used for determination of the magnetic fields from Balmer lines. source the X-ray brightest in our sample. No indicator for the presence of a hidden companion has been found. The magnetic field measurement yielded no detection.

- HD 186219: is listed as an A4III star in the SIMBAD cata$\log$. The X-ray source is separated from the optical position of the star by $12 \mathrm{arcsec}$. Its luminosity is $1.1 \times 10^{29} \mathrm{erg} / \mathrm{s}$ in the RASS catalog. The radial velocity is constant and no other indicator for a hidden companion has been found. No magnetic field has been detected in our observations.

- HD 215789: is an A3V star with a debris disk. A faint $\mathrm{X}$-ray source with a luminosity of $1.3 \times 10^{28} \mathrm{erg} / \mathrm{s}$ was detected near the optical position of the star in the PSPC pointing observations, separated from the star by 8 arcsec. Further observations of this object have been carried out with XMM-Newton, showing a moderately active X-ray source with flaring in one of the three observations. With plasma temperatures ranging between 2 and $8 \mathrm{MK}$ in a two temperature model for the less active state and up to 15 million Kelvin in the observation with the flare, this X-ray emission is in good agreement with a moderately active late-type companion. The X-ray luminosity varies between $6 \times 10^{27}$ and $3 \times 10^{28} \mathrm{erg} / \mathrm{s}$ and is therefor consistent with the ROSAT observations. Our own measurements showed no significant magnetic field.
- HD 217186: this bona fide single star of spectral type A1V is associated with an X-ray source observed in the PSPC pointing mode. The luminosity of the source is $9 \times 10^{28} \mathrm{erg} / \mathrm{s}$ and it is separated from the star by 15 arcsec. Our magnetic field measurements yielded weak signs of a longitudinal field of $97 \pm 53 \mathrm{G}$ in all lines and $102 \pm 70 \mathrm{G}$ using just the hydrogen lines, thus slightly below the $2 \sigma$ level.

- HD 224361: is listed in the SIMBAD database as an A1IV star according to Houk \& Cowley (1975). Other authors mention this star as spectral type A2p, which could indicate the presence of a magnetic field, but our measurements detected no magnetic field. Close inspection on the other hand shows a clear feature in the $\mathrm{H}_{\delta}$ line (see Fig. 7). The associated X-ray source was detected in the RASS and has a luminosity of $3.1 \times 10^{29} \mathrm{erg} / \mathrm{s}$. Its separation from the optical position of the star is 15 arcsec. No indicator for the presence of a hidden companion has been found.

- HD 224392: this A1V star is another IR excess star. The magnetic field measurements revealed a longitudinal field at a significance level of $2.5 \sigma$ using all lines. The field strengths in all and just the hydrogen lines are $134 \pm 51 \mathrm{G}$ and $89 \pm$ $60 \mathrm{G}$, respectively.Observations done during the RASS detected an X-ray source with a separation of 16 arcsec from the optical position of the A-type star and luminosity of $1 \times 10^{29} \mathrm{erg} / \mathrm{s}$. 


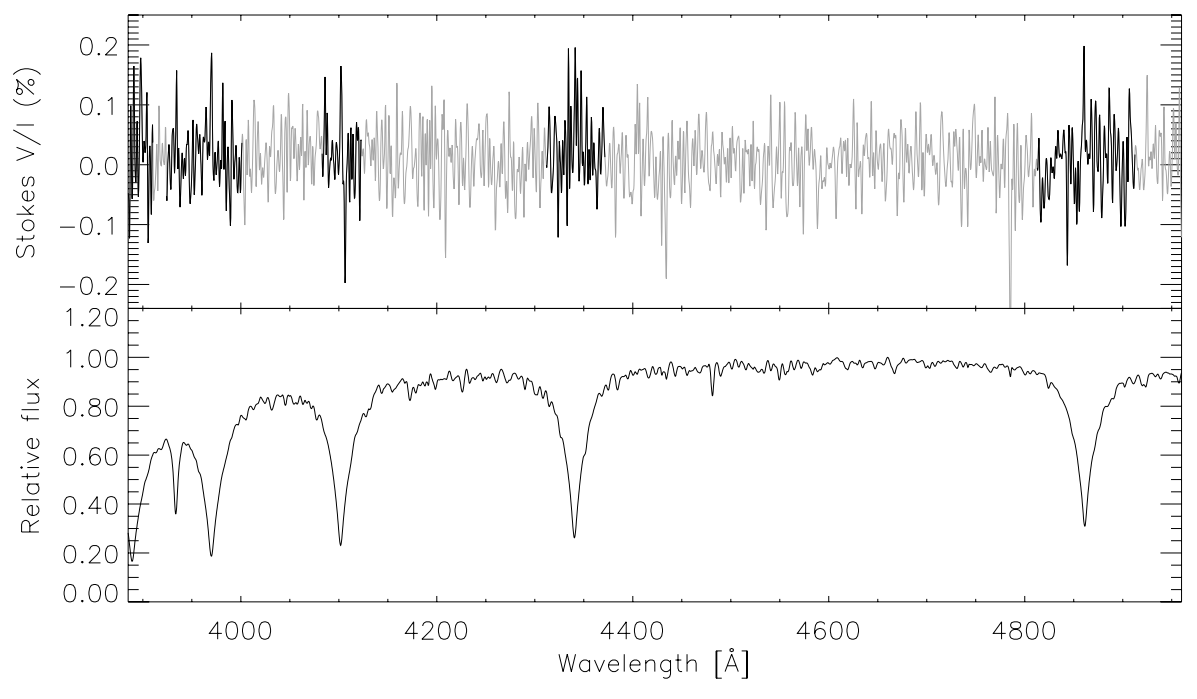

Fig. 7. Stokes $V / I$ spectrum and relative flux of the A1IV star HD 224361. The black areas in the Stokes $V$ spectrum indicate the regions used for determination of the magnetic fields from Balmer lines. The $\mathrm{H}_{\beta}$ and $\mathrm{H}_{\delta}$ lines show a clear Zeeman feature.
- HD 201601: the strongly magnetic rapidly oscillating A9p star with a rotation period of approximately $77 \mathrm{yr}$ has been selected to check that the instrument and data reduction were functioning properly. The measured values of the magnetic field using hydrogen lines agree well with the observations obtained a few years ago with the same instrument by Hubrig et al. (2004a). The calculated averaged quadratic magnetic field from the 18 measurements presented in that paper is $1085 \pm 56 \mathrm{G}$.

\section{Discussion and summary}

In this paper we present measurements of the longitudinal magnetic field in A-type stars obtained with the FORS1 instrument at the VLT Kueyen. The 13 observed A-type stars were taken from a list of single or resolved binary stars that are associated with an ROSAT X-ray source (Schröder \& Schmitt 2007) in order to test the hypothesis that X-ray emission in A-type stars might be related to the presence of magnetic fields. Two of our targets, HD 147084 and HD 148898, had been previously checked for magnetic fields, but the results of those observations lead to somewhat unsatisfactory significance levels of $1.5 \sigma$. With our measurements we obtained detections above the $3 \sigma$ level for HD 147084 and HD 148898, thus confirming previous measurements with low significance, and for HD 159312, which is an entirely new result. Our measurements for four additional stars, HD 174240, HD 186219, HD 217186 and HD 224392, yielded detections at a significance level between 2 and $3 \sigma$; we therefore consider these stars to be possible magnetic field candidates, which ought to be followed up. In the other six stars in our sample magnetic fields were not detected by our methods, although Zeeman features might possibly be present in some of the Balmer lines, warranting more sensitive observations for those stars.

With our observations we tested to what extent the magnetically confined stellar wind model (MCWS) proposed by Babel \& Montmerle (1997) can explain the observed X-ray emission of late B- and A-type stars. Using radiatively driven wind models and including the effect of magnetic confinement of the wind, Babel \& Montmerle (1997) were able to calculate a physical model explaining X-ray emission of the chemically peculiar A-type star IQ Aur. The basic assumption of the model is that the stellar wind is confined by the magnetic field lines. Due to the dipole structure of the field, the winds originating from the two different polar regions collide in the magnetic equatorial plane and produce shocks. In these shocks the kinetic energy of the wind is dissipated to eventually produce X-ray emission. In their calculations, Babel \& Montmerle (1997) find that the X-ray luminosity $L_{\mathrm{x}}$ scales approximatively like the mass loss rate $\dot{M}$, the wind velocity $v_{\infty}$ and the magnetic field $B$. The resulting scaling law for the X-ray luminosity is

$L_{\mathrm{x}} \simeq 2.6 \times 10^{30} \frac{\mathrm{erg}}{\mathrm{s}}\left(\frac{B_{*}}{1 \mathrm{kG}}\right)^{0.4} \xi$

where $\xi$ is described by

$\xi=\left(\frac{\dot{M}}{10^{-10} M_{\odot} / \mathrm{yr}}\right)^{\delta}\left(\frac{v_{\infty}}{10^{3} \mathrm{~km} \mathrm{~s}^{-1}}\right)^{\epsilon}$

Based on the calculations, $\delta$ and $\epsilon$ can be obtained as $\simeq 1$ and $1-1.3$ respectively. The X-ray observations of IQ Aur can be understood with a wind velocity of IQ Aur of 500-900 km s${ }^{-1}$ and mass loss rates of $4.5 \times 10^{-11} M_{\odot} /$ yr. Czesla \& Schmitt (2007) studied magnetic late B- and A-type stars that have been observed in X-rays with the Chandra telescope and found values ranging from $860-940 \mathrm{~km} \mathrm{~s}^{-1}$ for $v_{\infty}$ and $1-4 \times 10^{-11} M_{\odot} / \mathrm{yr}$ for $\dot{M}$ to account for the observed X-ray emission within the framework of the MCWS model. We use these values to estimate the X-ray emission from those of our stars with magnetic fields measured at a $3 \sigma$ level, noting, however, that the uncertainties on the wind properties of up to one order of magnitude only allow a rough estimate of the expected X-ray emission.

The magnetically confined wind shock model predicts an X-ray luminosity of $5 \times 10^{29} \mathrm{erg} / \mathrm{s}$ with values of $900 \mathrm{~km} \mathrm{~s}^{-1}$ for the wind velocity $v_{\infty}, 4 \times 10^{-11} M_{\odot} / \mathrm{yr}$ for $\dot{M}$ and $240 \mathrm{G}$ for $B$ in the case of HD 159312 (see Fig. 5). Decreasing the mass loss rate to $1 \times 10^{-11} M_{\odot} /$ yr lowers the predicted X-ray luminosity to $1.3 \times 10^{29} \mathrm{erg} / \mathrm{s}$. The observed X-ray luminosity for HD 159312 in the PSPC pointing and the ROSAT All-Sky Survey observations is $9.1 \times 10^{29} \mathrm{erg} / \mathrm{s}$, hence of the same order of magnitude as the calculated value.

The magnetic field in HD 148898 is slightly weaker than in the previous object HD 159312, resulting in a lower calculated X-ray luminosity. Assessing the values derived by Czesla \& Schmitt (2007) and Babel \& Montmerle (1997), the wind shock model predicts an $L_{\mathrm{x}}$ of $1-4 \times 10^{29} \mathrm{erg} / \mathrm{s}$ for mass loss rates of $2-4 \times 10^{-11} M_{\odot} / \mathrm{yr}$ and wind velocities of $500-900 \mathrm{~km} \mathrm{~s}^{-1}$. The observed X-ray luminosity is $1 \times 10^{29} \mathrm{erg} / \mathrm{s}$, therefore in good 
accordance with the predicted values for low wind velocities and mass loss rates.

In the case of HD 147084, which is listed in the SIMBAD database as a (bright) giant A4 star, the situation is a bit more complicated (see Sect. 3.3). HD 147084 might be a member of the group of Herbig Ae/Be stars (Herbig 1960), whose X-ray emission has been studied by various authors (Hamaguchi et al. 2005; Stelzer et al. 2006b; Zinnecker \& Preibisch 1994; Damiani et al. 1994). Another possible explanation for the high luminosity of this star is that it is an evolved massive star in the Cepheid phase, whose radial pulsations might be able to explain the variations in the radial velocity. In both scenarios, the observed X-ray emission is not unexpected.

In our test for a correlation between the X-ray luminosity of the associated X-ray source and the measured magnetic field strength we found no clear dependence between the two values. The X-ray brightest star, HD 182761, has no detectable magnetic field. To reproduce the X-ray luminosity of $1.2 \times 10^{30}$ with the magnetically confined wind shock model, this star would require an easily detectable magnetic field of $1.5 \mathrm{kG}$, assuming the wind velocity and mass loss rate given in Sect. 3.2. Therefore, HD 182761 either has unusual values for wind velocity and mass loss rates, or an explanation for its X-ray emission must be sought outside the framework of the MCWS theory.

On the other hand, those stars with a detected magnetic field possess X-ray emission are in agreement with the predicted values from the MCWS model of Babel and Montmerle. In this context however, the question arises of why the standard star HD 201601, a chemically peculiar A9 star with a magnetic field above $1 \mathrm{kG}$, was not detected in X-rays. A possible explanation might be that the early A-type stars, IQ Aur is an A0p star, still possess a weak wind, which is necessary to produce the X-ray emission, but the later A-type star lack such a wind. In addition, the star HD 201601 is rather old and has already spent more than $60 \%$ of its life on the main sequence (Hubrig et al. 2000), which might explain the absence of X-ray activity in this star with a strong magnetic field.

The results in this paper agree with the conclusions Czesla \& Schmitt (2007) presented in their analysis of A- and B-type stars with substantial magnetic fields. They too found that the presence of a (strong) magnetic field is not necessarily accompanied by a strong X-ray emission. Other parameters like the mass loss rate and the wind velocity may play an even more important role.

Thus 7 out of 13 studied stars show (weak) signs of magnetic fields, yet only in three of them these features were strong enough to be detected at a $3 \sigma$ level. Even in some of the undetected 6 stars, e.g. HD 163336 and HD 224361, there were increased signals in the Stokes $V / I$ spectra near some of the Balmer lines. Since the Zeeman features can only be explained by the presence of a magnetic field, more accurate observations over the rotation periods of the studied stars are needed to confirm the existence of those fields.

\section{References}

Babel, J., \& Montmerle, T. 1997, A\&A, 323, 121

Bagnulo, S., Szeifert, T., Wade, G. A., Landstreet, J. D., \& Mathys, G. 2002, A\&A, 389, 191

Berghöfer, T. W., \& Schmitt, J. H. M. M. 1994, A\&A, 292, L5

Borra, E. F., \& Landstreet, J. D. 1980, ApJS, 42, 421

Bychkov, V. D., Bychkova, L. V., \& Madej, J. 2003, VizieR Online Data Catalog, 340, 70631

Czesla, S., \& Schmitt, J. H. H. M. 2007, A\&A, 465, 493

Damiani, F., Micela, G., Sciortino, S., \& Harnden, Jr., F. R. 1994, ApJ, 436, 807 Donati, J.-F., Babel, J., Harries, T. J., et al. 2002, MNRAS, 333, 55

Eggen, O. J. 1998, AJ, 116, 1314

Feldmeier, A., Puls, J., \& Pauldrach, A. W. A. 1997, A\&A, 322, 878

Gagne, M., Caillault, J.-P., Stauffer, J. R., \& Linsky, J. L. 1997, ApJ, 478, L87

Gagné, M., Oksala, M. E., Cohen, D. H., et al. 2005, ApJ, 628, 986

Groote, D., \& Schmitt, J. H. M. M. 2004, A\&A, 418, 235

Hamaguchi, K., Yamauchi, S., \& Koyama, K. 2005, ApJ, 618, 360

Harnden, Jr., F. R., Branduardi, G., Gorenstein, P., et al. 1979, ApJ, 234, L51

Herbig, G. H. 1960, ApJ, 131, 632

Houk, N., \& Cowley, A. P. 1975, Michigan Catalogue of two-dimensional spectral types for the HD star (Ann Arbor: University of Michigan, Departement of Astronomy)

Hubrig, S., North, P., \& Mathys, G. 2000, ApJ, 539, 352

Hubrig, S., Le Mignant, D., North, P., \& Krautter, J. 2001, A\&A, 372, 152

Hubrig, S., Bagnulo, S., Kurtz, D. W., et al. 2003, in ASP Conf. Ser. 305, ed. L. A. Balona, H. F. Henrichs, \& R. Medupe, 114

Hubrig, S., Kurtz, D. W., Bagnulo, S., et al. 2004a, A\&A, 415, 661

Hubrig, S., Schöller, M., \& Yudin, R. V. 2004b, A\&A, 428, L1

Hubrig, S., North, P., Schöller, M., \& Mathys, G. 2006, Astron. Nachr., 327, 289

Hubrig, S., North, P., \& Schöller, M. 2007a, Astron. Nachr., 328, 475

Hubrig, S., Pogodin, M. A., Yudin, R. V., Schöller, M., \& Schnerr, R. S. 2007b, A\&A, 463, 1039

Kramer, R. H., Cohen, D. H., \& Owocki, S. P. 2003, ApJ, 592, 532

Landstreet, J. D. 1982, ApJ, 258, 639

Levato, H., Malaroda, S., Morrell, N., \& Solivella, G. 1987, ApJS, 64, 487

Lucy, L. B. 1982, ApJ, 255, 286

Lucy, L. B., \& White, R. L. 1980, ApJ, 241, 300

Owocki, S. P., \& Cohen, D. H. 1999, ApJ, 520, 833

Schmitt, J. H. M. M., \& Kürster, M. 1993, Science, 262, 215

Schmitt, J. H. M. M., \& Liefke, C. 2004, A\&A, 417, 651

Schmitt, J. H. M. M., Fleming, T. A., \& Giampapa, M. S. 1995, ApJ, 450, 392

Schmitt, J. H. M. M., Groote, D., \& Czesla, S. 2005, in 13th Cambridge Workshop on Cool Stars, Stellar Systems and the Sun, ed. F. Favata et al., ESA SP-560, 943

Schröder, C., \& Schmitt, J. H. M. M. 2007, A\&A, 475, 677

Seward, F. D., Forman, W. R., Giacconi, R., et al. 1979, ApJ, 234, L55

Stelzer, B., Huélamo, N., Micela, G., \& Hubrig, S. 2006a, A\&A, 452, 1001

Stelzer, B., Micela, G., Hamaguchi, K., \& Schmitt, J. H. M. M. 2006b, A\&A, 457, 223

Townsend, R. H. D., Owocki, S. P., \& Ud-Doula, A. 2007, MNRAS, 978 ud-Doula, A., \& Owocki, S. P. 2002, ApJ, 576, 413

ud-Doula, A., Townsend, R. H. D., \& Owocki, S. P. 2006, ApJ, 640, L191

Zinnecker, H., \& Preibisch, T. 1994, A\&A, 292, 152 